\title{
Research Collaborations and Quality in Research: Foes or Friends?
}

\author{
Elisabetta Vaudano
}

\section{Contents}

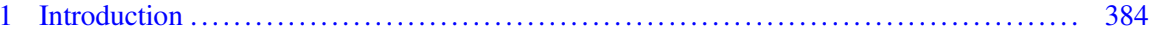

2 Successful Collaborative Research and High Research Quality Are Interdependent ..... 385

3 Quality of Research and Sustainability .................................. 388

4 The Importance of Effective Governance in Collaborative Research ................. 389

5 On Data Sharing, Collaborative Research and Research Quality .................... 390

6 Enlarging the Collaborative Research Environment: Regulators and Patients as Important Partners for Research Quality ........................................... 393

7 Why Scientists Should Consider Quality as a Key Parameter for Their Collaborative Research from the Very Start ........................................... 394

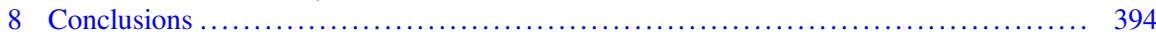

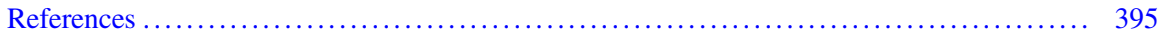

\begin{abstract}
Collaboration is the cornerstone of nowadays research. Successful collaborative research and high research quality go hand in hand. Collaborative research needs to build on common and upfront expectations for the quality of its outputs. This is necessary to enable a trustful research environment where all are committed to contribute and can share the rewards. A governance and leadership are critical for this to happen as well as a policy for openness and for effective data sharing. Collaborative research is often large-scale research: to be successful it needs good research practice as an enabler. Collaborative projects are ideal vehicles to promote high research quality, among other by enabling the delivery of results of high external validity and the development and implementation of standards. Robustness of results increases when confirmed by combining different methods and tools and even more when results are obtained while sharing and learning different approaches and languages of science. When doing collaborative
\end{abstract}

E. Vaudano $(\square)$

The Innovative Medicines Initiative, Brussels, Belgium

e-mail: elisabetta.vaudano@imi.europa.eu 
research, there is the best opportunity to combine the different experience and expertise of all partners by design to create a more efficient and effective environment conductive for high-quality research. Using as example the publicprivate partnership type of projects created by the Innovative Medicines Initiative, the chapter covers the key aspects of the complex relationship between collaborative research and quality of research providing insights on the critical factors for delivering both a successful collaboration in research and robust highquality research outputs.

\section{Keywords}

Data quality · Governance $\cdot$ Industry $\cdot$ Knowledge $\cdot$ Research output ·

Stakeholders · Trust

\section{Introduction}

Most modern biomedical research depends on collaboration. While research collaboration is usually thought to mean an equal partnership between two or more members who are pursuing mutually interesting and beneficial research, research collaborations come in many types and species where the relationship between the collaborators varies both in its depth and in the balance of the relationship.

At the "light" end, research collaboration might simply imply getting access to assets such as reagents, instruments, assays and data, owned by one researcher or by another researcher for its own research interests. A rather different type of collaboration is contract work where one, often private, entity enters into contractual relationships with another, often academic, institution to get a certain type of research work done. On the "heavy" end, we then have fully collaborative research where research is carried out jointly from idea generation to its implementation.

Most collaborative biomedical research occurs between two or more academic institutions. When the focus of research moves from basic to more applied research, its scope requires often involving actors beyond academia. A special type of collaboration is that between public and private institutions in public-private partnerships, such those created by the Europeans, the Innovative Medicines Initiative (IMI) (The Innovative Medicines Initiative 2009). The IMI is a joint initiative (public-private partnership) of the European Commission (EC) and the European Federation of Pharmaceutical Industries and Associations (EFPIA). The current programme, IMI2 Joint Undertaking (IMI2 JU), is part of the Horizon 2020 Framework programme. IMI fosters collaborative research among many different stakeholders, from large industries (mainly biopharmaceutical but most recently also from diagnostics, information technology and imaging industries) to small medium-sized enterprises, academia, hospitals, regulators, patient organisations, etc. These partnerships (consortia) implement a research work plan where all members work jointly towards agreed and fixed objectives and deliverables. The resources (often very significant) are provided, on one side, by funding from the EC to all eligible entities and, on the other side, through in-kind contributions from the industry members of the EFPIA and in some projects by Associated Partners to the 
IMI2 JU. Importantly, neither the EFPIA members nor the IMI2 JU Associated Partners are eligible for funding. Thus, they participate at own cost and provide resources (experts' time, setting and running of assays and models, clinical studies, etc.) for the implementation of the research. The IMI has proven to be a very successful collaboration model with significant output already delivered (Faure et al. 2018). The terms of the collaboration in the IMI consortia are established by the grant agreement that all partners have to sign with the IMI and, most importantly, by the consortium agreement that all members of the consortium have to sign among themselves.

A lot has been written regarding the pros and cons of research collaboration and on the "added value" of research collaboration. Here, I would like to focus on the complex and important relation between research done collaboratively and quality of its (research) output. The aim is not to demonstrate any potential or even causal relationship but to bring to the attention of the reader the important interdependences among collaborative research and research quality, highlighting benefits and challenges.

To provide some "real-life" evidence, I will use as examples cases of collaborative research in public-private partnerships of the type created by IMI. The IMI research collaborations are highly complex, in terms of both content and stakeholders involved, and the robustness of the research outputs is affected by all factors that in isolation may be relevant for "simpler" research collaborations. At a recent event for the 10th year anniversary of the IMI, the Commissioner Moedas defined the research collaboration fostered by IMI as "radical collaboration" (The Innovative Medicines Initiative Radical Collaborations 2018).

\section{Successful Collaborative Research and High Research Quality Are Interdependent}

High-quality research produces results that can be confidently used as the basis for generating new knowledge or for application purposes (e.g. the development of a new drug). When choosing collaborators the trust in the quality of their research outputs is a key factor. Thus high-quality research and successful collaborative research go hand in hand. Robustness of results is increased when it can be confirmed by combining different methods and tools and even more when results are obtained while sharing and learning different approaches and languages of science. When doing collaborative research, there is the best opportunity to combine the different experiences and expertise of all partners by design to create a more efficient and effective environment conductive for high-quality research.

Most IMI consortia perform collaborative research that is highly multidisciplinary and where research teams work jointly across the public and private sector. For example, the IMI "Methods for systematic next generation oncology biomarker development", ONCOTRACK project (Methods for Systematic Next Generation Oncology Biomarker Development ONcoTRACK Project 2016), is a precompetitive research project that was created to tackle the general problem of 
identification and validation of clinically robust biomarkers in oncology (Schütte et al. 2017). The project team included eight EFPIA industry teams, nine universities/research institutions and four SMEs. Among the outputs of this consortium is a high-throughput screening platform for three-dimensional patient-derived colon cancer organoid cultures. The platform has been fully validated for assay robustness and reproducibility, an achievement only possible via the collaborative efforts of the multidisciplinary industry-academia team, built on a foundation of trust in the quality of the work of each partner with criteria agreed and applied from the very beginning of the partnership (Boehnke et al. 2016).

However, such complex cross-fertilisation comes with some caveats. It must not be underestimated that, for such collaborative efforts to succeed, all partners need time and good will to adjust to each other's way of working and thinking. Technical jargon can be very different; the same acronym may have very different meanings (e.g. API: "Active pharmaceutical ingredient" or "application programming interface"). The time and resources necessary for this preparatory work should be considered carefully with attention to aspects such as a good communication platform and legal support. It can be a lengthy and challenging process: timelines should be adjusted accordingly, and expectations!

Trust is the uppermost key factor of success for a research collaboration. In a collaboration, researchers depend for success on both their own results and those of their partners. Since it might be challenging and impracticable to share fully details of work going on in different laboratories, the application of high-quality standards, understood and agreed by all partners from the very start of the collaborative work, is an important enabler for a successful partnership. Once such standards are in place, there is higher motivation and opportunity for the achievement of robust high-quality results and conclusions.

Collaborative research has enabled research programmes at an unprecedented scale. Typical examples are those from genetic research. Here, large-scale collaborative genome studies have delivered huge amount of data opening new avenues for the understanding of disease biology via the use and reuse of these data by many scientists. A stringent adherence to good research practice and quality control is necessary when working at such scale. Among the many programmes is worth mentioning the "Encyclopedia of DNA Elements, ENCODE initiative funded by the National Human Genome Research Institute" (Encyclopedia of DNA Elements (ENCODE) 2019). ENCODE aims to identify all functional elements in the human and mouse genomes and make them available through the project's freely accessible database. The ENCODE project has developed standards for each experiment type to ensure high-quality, reproducible data and novel algorithms to facilitate analysis (ENCODE Consortium 2017). As a result of outreach and collaboration, enabled by such quality-driven approach, ENCODE has been highly successful, and its data are widely used to deliver high-quality publications.

While standards are needed to deliver a successful collaboration, conversely collaborative research can be a powerful tool to boost the development and implementation of standards and interoperability of results, which again significantly enhances research quality as shown by two further examples below. 
Modelling and simulation (M\&S), a technology providing the basis for informed, quantitative decision-making, is of high importance in modern drug development. A lack of common tools, languages and ontologies for $M \& S$ often leads to inefficient reuse of data and duplication of effort by academic, industrial and regulatory stakeholders, as well as hindering research quality. The IMI "Drug Disease Model Resources", DDMoRe (Drug Disease Model Resources DDMoRe Project 2012), consortium delivered a set of integrated tools, exchange standards and training to improve the quality and cost-effectiveness of model-informed decision-making for pharmaceutical research and development. The set of standards has been designed both for model and workflow encoding and for storage and transfer of models and associated metadata. One of the key products the project developed based on these standards is the publicly available DDMoRe model repository (DDMoRe Model Repository 2017). It provides access to more than 100 annotated and "ready to use" pharmacokinetic (PK), pharmacodynamic (PD), PK/PD, physiologically based PK (PBPK), statistical and systems biology models applied in different therapeutic areas like oncology, diabetes and neuroscience. The model repository content is quality assured by experts from the DDMoRe model review group, who provides on-demand impartial review and assesses the model's technical validity and reproducibility. The models that pass the review are certified and can be confidently reused by anyone either commercially or for research purposes.

Rheumatoid arthritis (RA) is a very common and debilitating condition due to many underlying disease mechanisms, thus the plethora of animal models of which the translatability and reproducibility is not well established. The IMI "Be The Cure" BTCure ("Be The Cure" BTCure Project 2012) consortium developed an infrastructure to standardise procedures to generate and interpret commonly used RA animal models, as well as to generate new types of RA animal models. Their work has shown how data obtained from these models might lack quality and reproducibility, due to insufficient documentation and nomenclature, wrong presentation of results and data, as well as the selection of inappropriate models and strains. Most importantly, the BTCure team proposed relevant solutions and developed training material to improve the quality of RA models (Holmdahl 2015). The achievement has been possible only by the joint collaborative work of key opinion leaders in the field of RA to build a critical mass of experts and achieve consensus via a series of dedicated workshops (e.g. BTCure Consortium 2012).

High-quality research has external validity. This does not mean its results are fully reproducible in an identical manner anywhere and anytime but that each result comes together with the awareness and understanding on the potential contextual factors that determine the variation over space or time. Collaborative research enables several partners in a consortium to replicate each other results and then share the obtained knowledge. This dramatically increases the robustness of the research outputs allowing to understand factors impinging on reproducibility of results.

For example, drug-induced liver injury (DILI) is a serious issue not only for patients and health-care professionals but also for the pharmaceutical industry and regulatory authorities. Human-specific and idiosyncratic adverse reactions are often 
detected only at the clinical and post-marketing stages leading to costly termination of drug development and risk for the patients with black box warnings or even withdrawal of drugs from the market. DILI remains a significant problem in drug development, suggesting that currently used in vitro models are not appropriate for effective screening. The IMI "Mechanism-Based Integrated Systems for the Prediction of Drug-Induced Liver Injury" MIP-DILI (Mechanism-Based Integrated Systems for the Prediction of Drug-Induced Liver Injury MIP-DILI Project 2012) consortium has run a comprehensive, multicentre, unbiased assessment to test this unequivocally. The consortium used a panel of compounds implicated in DILI in man, in order to determine whether any of these simple cell models per se are actually predictive of human DILI. Furthermore, by using a small panel of DILIand non-DILI-implicated compounds and basic measures of cell health, it monitored reproducibility across different sites, thereby ensuring that data should be more definitive than any currently available (Sison-Young et al. 2017).

Multicentre collaborations can expose systematic biases and identify critical factors to be standardised. Human-induced pluripotent stem cells (iPSCs) are powerful tools for novel in vitro models in basic science and drug discovery. iPSCs need to be differentiated using lengthy complex procedures with increased possibility for variability and noise in the results. The IMI "Stem cells for biological assays of novel drugs and predictive toxicology" (STEMBANCC) project (Stem Cells for Biological Assays of Novel Drugs and Predictive Toxicology Project 2014) runs a unique assessment of the inter- and intra-laboratory reproducibility of transcriptomic and proteomic read-outs using two iPSC lines at five independent laboratories in parallel. By achieving larger sample numbers in a collaborative approach with crosslaboratory studies, the team could detect identifiable sources of variation that investigators can control. This study also strongly advocates for transparency via disclosure of identified variation-inflating confounders in published iPSC differentiation protocols (Volpato et al. 2018).

\section{Quality of Research and Sustainability}

Collaborative research connects researchers and institutions. The created networks are key assets that go beyond the lifetime of an individual project and can play an important function as ambassadors of the culture of quality in research. The networks can lead by example but can only be successful in time by being open to new members and by including elements of education, training and dissemination of good practice and processes.

The IMI "Combatting Bacterial Resistance in Europe" (COMBACTE) consortium ("Combatting Bacterial Resistance in Europe" COMBACTE Project 2015) has delivered a high-quality European clinical trial and laboratory network in which new antibacterial drugs can be evaluated for the treatment and prevention of infections caused by multiresistant bacteria. Over 650 laboratories and over 850 clinical trial sites all over Europe are already interconnected. Development of new antibiotics is hampered by limited market incentives but also significantly limited by suboptimal 
quality of trial results and lack of innovative methods robust enough to be accepted by the regulators. The COMBACTE network of statistical experts "STAT-Net" has reviewed and tested several innovative trial designs and analytical methods for randomised clinical trials, which has resulted in eight recommendations made available to the community in a white paper (de Kraker et al. 2018). The COMBACTE project has been one of the first of the IMI programme New Drugs 4 Bad Bugs (ND4BB), which today represents an unprecedented partnership between industry, academia and biotech organisations to combat antimicrobial resistance in Europe. The EUR 650 million programme comprises currently eight projects and has built the foundation for the IMI2 Accelerator programme.

Collaborative networks with a proven record of high-quality research increase their opportunities for further collaboration and funding, thus becoming sustained. A very fitting example is that of the IMI consortium "European Autism Interventions A Multicentre Study for Developing New Medications", EU-AIMS (European Autism Interventions - A Multicentre Study for Developing New Medications Project EU-AIMS 2012), identifying markers of autism that would help in earlier and more accurate diagnosis, prognosis and the development of new therapies. The project applied the highest standard of research quality, which among others led to five "letters of support" from the European Medicines Agency (EMA) (European Medicines Agency 2015). These recognised results are the foundations of the follow-up project "Autism Innovative Medicine Studies-2-Trials", AIMS-2-Trials (Autism Innovative Medicine Studies-2-Trials AIMS-2-Trials Project 2018), which is a 115 million EUR cross-Atlantic collaborative initiative, nearly four times the resources of EU-AIMS.

\section{$4 \quad$ The Importance of Effective Governance in Collaborative Research}

Not all collaborations are successful in delivering high-quality research. For having a positive impact on quality of research, a collaboration must be based on transparency among all involved stakeholders, meaning clearly established governance rules for the implementation of the research work plan and sharing of knowledge thereof. Rules have to be in place from the very start of the collaboration, be communicated to all collaborators and be agile and not overly complicated, to avoid being seen as a burden instead of an enabler. An efficient governance is necessary to ensure good working relationships in the collaboration, a sense of high degree of accountability in all partners and spirit of joint responsibility while also keeping expectations realistic. Governance has to provide leadership for the collaborator's team and create a "trust environment" which in the case of the most complex partnerships might need a third party to act as a neutral broker, like in the case of the IMI Programme Office (Goldman 2012).

The collaboration governance must guarantee respect for the rights of all collaborators and that the collaboration will be rewarding for all involved. This latter can be seen as a challenge when trying to balance, on one side, the need for 
open innovation and knowledge sharing and, on the other, the need for intellectual property rights (IPRs) to be protected. A one-size-fits-all solution does not exist for all types of research collaborations, but some principles can be generally applicable. First, there needs to be a built-in degree of flexibility when establishing the rules for IPRs, with opportunity for adaptation with the development of the research programme. Secondly, IPR agreements must be agreed before the collaboration starts, providing consortia with less legal uncertainty and avoiding useless a posteriori discussions. Most importantly legal agreements cannot and must not substitute a well-thought-through high-quality research programme. All partners have to have a clear understanding about their own knowledge/data/assets that they need to share with the other collaborators for the implementation of a highquality research programme and achievement of its objectives. These objectives and the pathway to achieve them have to be clear and sound.

To achieve impact and promote sustainability, all partners have to agree on the sharing of the research outputs among themselves and with third parties. In the IMI programme, all consortium members sign a consortium agreement before the start of the activities which details the collaboration rules of play and governance. A template prepared by EFPIA shows what a consortium agreement might look like (European Federation of Pharmaceutical Industries and Associations 2015). In addition, the IMI Programme Office plays a neutral role and offers impartial advice to all partners during negotiations on IPRs. This process and support ensure that the resulting agreement is in line with the IMI IPRs provisions and does not leave some project partners at a disadvantage (Laverty and Poinot 2014).

A good governance structure is important in collaborative research but is not sufficient for success. It has to be mentioned that collaborative research can still be silos research, where the collaborators share a pot of funding but otherwise work mainly independently just continuing and expanding their own internal research programme. Such "collaborations" represent a missed opportunity for crossfertilisation and learnings and thus are not very inductive to research quality. In my own experience, these are also the consortia where most IPR-related issues arise and the signature of the consortium agreement might be delayed by lengthy, often circular, discussions.

\section{On Data Sharing, Collaborative Research and Research Quality}

Reluctance to data sharing has been shown to correlate with poor quality of the research results; see, for example, the paper by Wicherts et al. (Wicherts et al. 2011). Reasons for not sharing data range from lack of time and resources (Tenopir et al. 2011) to legal issues and fear for data misuse (Majumder et al. 2016; Parse Insight Consortium 2019).

Still, especially in collaborative research, data sharing is a fundamental factor for the delivery of high-quality research. It is essential for increasing reproducibility and transparency in research. This has been now recognised by publishers, by major 
research organisations and by funders (e.g. the Transparency and Openness Promotion guidelines (Nosek et al. 2015), a recent Nature Editorial (Nature Editorial 2017) and the OpenAIRE initiative of the European Framework Programme H2020 (European Commission 2014)).

Data sharing has to be enabled by the upfront creation of a framework that is acceptable and accepted by all partners, which is not always a simple endeavour. The IMI "Diabetes Research on patient stratification", DIRECT (Diabetes Research on Patient Stratification DIRECT Project 2013), consortium has shared its experience and learnings on how to develop a governance for data sharing in their collaboration in a recent paper published in Life Sciences, Society and Policy (Teare et al. 2018). What they show is that designing an internal governance structure to oversee access to a centralised database for research purposes can be a time-consuming and politically sensitive process. Partners might have different expectations and requirements for taking part. Moreover, especially for international collaborations, it might be tricky to provide a framework that is acceptable legally and ethically across borders and still is sufficiently speedy to allow research.

However, several solutions are emerging to address these concerns and enable data sharing, such as those developed by the IMI "European Medical Information Framework", EMIF, project (Trifan et al. 2018) and the Cohen Veterans Bioscience Brain Commons (Grossman 2017). Another useful resource to help the research community in the sharing of data and samples has been delivered by partners from the IMI BTCure consortium jointly with other experts from around the world. The "International Charter of principles for sharing bio-specimens and data" addresses the points necessary to enable effective and transparent data and samples sharing and even provides a general template for data and material sharing (Mascalzoni et al. 2015).

Once data sharing is enabled, it delivers significant benefits for both those sharing and the scientific community at large and is an important first step for sustainability of results. This can be exemplified by the IMI "Unrestricted leveraging of targets for research advancement and drug discovery”, ULTRADD (Unrestricted Leveraging of Targets for Research Advancement and Drug Discovery ULTRADD Project 2016), project data policy. ULTRADD aims to identify and validate under-explored protein targets by profiling target-directed chemical and antibody probes in patient-cellderived assays at the highest quality, providing biomarker and phenotypic read-outs in a more disease-relevant context. The consortium adopted a policy of full open access. The wider scientific community will therefore have ready access to much of the knowledge, data and tools generated by the project. True to its promise, the consortium has already made available to the broad community the first batch of high-quality datasets (Cell Assay Datasets 2017).

The sharing of high-quality data strongly facilitates moving research forward providing the bases for new applications and benefit to society. The IMI "Integrating bioinformatics and chemoinformatics approaches for the development of Expert systems allowing the in silico prediction of toxicities" (eTOX) consortium developed innovative strategies and novel software tools to better predict the safety and side effects of new candidate medicines for patients (Sanz et al. 2017). 
The achievements were enabled by a unique shared database based on high-quality legacy information provided by EFPIA companies from their own preclinical drug toxicity studies. By the end of the project, the database has information from over 8,000 toxicity studies on almost 2,000 compounds, of which around a fifth are approved drugs. Several of the in silico algorithms developed by eTOX are now used by the pharmaceutical industry for better prediction of potential drug toxicities.

Data and knowledge in order to be shareable need to be properly managed. This can be difficult for researchers working on their own which might lack the expertise, experience, technical solutions and resources for proper data and knowledge management (DKM) during and after a project. At IMI and elsewhere, research collaborations involve more and more experts and resourcing for DKM, which is a very positive trend. The inclusion of relevant provisions for DKM in any research collaboration will enhance the quality of the research output with a cascade effect for the quality of any future project that will build on such background. A good data management and data stewardship are fundamental for data quality. Science funders, publishers and governmental agencies are beginning to require data management and stewardship plans for data generated by their grantees (see, e.g. the open access and data management for projects of IMI (Open Access and Data Management for Projects 2015)). Findable, Accessible, Interoperable and Reusable (FAIR) data (Wilkinson et al. 2016) will become more attractive for reuse and the data generators sought for new collaborations, thus automatically ensuring the sustainability of their results. Making it convenient for scientists to describe, deposit and share their data and to access data from others, plus promulgating best data practices through education and awareness, will help the future of science as well as the future of data preservation.

The awareness of this problem led to the creation of the IMI "Delivering European Translational Information \& Knowledge Management Services" eTRIKS project. The eTRIKS delivered an open, sustainable research informatics and analytics platform for use by IMI (and other) projects with knowledge management needs. In addition, the project partners provide associated support, expertise and services to ensure users gain the maximum benefit from the platform. One important resource is the eTRIKS/ELIXIR-LU - IMI Data Catalogue (eTRIKS/ELIXIR-LU - IMI Data Catalogue 2015), a sustained a metadata repository linking the massive data available in a global system that can be optimally leveraged by scientists. Researchers can access the available data and are encouraged to add their data in the database to create awareness and recognition of their data contribution and demonstrate value of partner projects.

Despite the increased emphasis on the importance for DKM, often there is yet not enough awareness about the DKM needs and resources for a project. The gaps are identified too late, when activities are already up and running and all budget committed elsewhere; thus more attention is needed to this critical area in all its aspects, technical, ethical, societal and legal. 


\section{$6 \quad$ Enlarging the Collaborative Research Environment: Regulators and Patients as Important Partners for Research Quality}

For the outputs of a biomedical translational research collaboration to impact health care, the involvement with regulators and patients is an important success factor, not the least because of the positive influence on research quality.

A partnership with regulators increases the awareness and drive of a consortium for the highest-quality results. The involvement of regulators (and in some cases also health technology assessment bodies and payers) is of high benefit to ensure that the project output meets the required standards and is of quality good enough to be taken up and used in drug development or medical practice. For example, the IMI "Understanding chronic pain and improving its treatment" EUROPAIN consortium developed rigorous processes for quality assurance of centres using in a standardised way quantitative sensory testing (QST) for measuring pain in patients. This is a novel approach for stratification of patients in clinical trials for pain medicines (Baron et al. 2017; Vollert et al. 2015). EUROPAIN engaged with the European Medicines Agency (EMA) to discuss their approach and results (The Innovative Medicines Initiative 2017a), and their approaches were considered in the updated guidelines for the development of medicines for pain treatment (European Medicines Agency 2016).

The IMI actively encourages to involve regulators as early as possible in the research work plan and has published specific guidelines for consortia (The Innovative Medicines Initiative 2017b). Many IMI projects have benefited from such interaction (Goldman et al. 2015). Similar approach is that of the Accelerating Medicines Partnership (AMP) (Accelerating Medicines Partnership AMP 2014) where the Federal Drug Administration (FDA) is a formal partner and of the Critical Path Institute (C-Path). C-Path has a large portfolio of initiatives where scientific rigour is at the basis of consensus building among participating scientists from industry and academia and the FDA to deliver new drug discovery tools (DDTs) for a given use in product development (Critical Path Institute C-Path 2005). This combination of good research practice and regulatory acceptance has allowed many of C-Path FDA-qualified DDTs to become open standards for the scientific community.

Researchers are now well aware that patients bring unique knowledge and skills to projects, which can significantly help to improve the quality of research. An exemplar collaboration where patients have actively contributed to improve research quality is the IMI "Unbiased Biomarkers for the Prediction of Respiratory Disease Outcomes" (UBIOPRED) project. Patients played a big role in the project, including participation in the scientific and the ethics boards. They helped in many aspects of the project and contributed to the fine-tuning of the research protocols. The consortium has published a very useful guide for effective engagement of patients in biomedical research (Geissler et al. 2017). 


\section{$7 \quad$ Why Scientists Should Consider Quality as a Key Parameter for Their Collaborative Research from the Very Start}

As already stressed in the previous sections, there are multiple reasons why research quality and success in a research collaboration go hand in hand. When working as a consortium, researchers will create a collective reputation, dependencies between partners and shared ethical responsibilities. They will also have to agree in the sharing of resources and funding, which may be significant in amount and of high value from the financial and scientific point of view. It is at this very early stage that a collaborative team has to align the expectation for high research quality. To achieve impact and success in their collaborative research, scientists need to get to a common understanding and agreement on how they will deliver high-quality results ideally taking advantage of common standards and guidance when available. It is very important that this occurs upfront, before picking up the pen for writing the first line of the joint grant. In fact, it is not possible to "fix" the quality of results once they have been already delivered; this will have only a "cosmetic" effect that will be detrimental both for those producing the results and for those that will base their independent work on such evidence. Results that will not be used or will not be found useful because of poor quality will be wasted and a waste of the public or private funding. Thus, there is an important ethical value in thriving for research quality and for doing so from the very beginning.

In the world of collaborative research, reputation is of paramount importance as well as trust. If a researcher delivers results of poor quality, this will influence strongly and negatively her/his reputation. In a consortium, this might affect the reputation of everybody. Delivering poor-quality results might generate mistrust in a collaboration and thus jeopardise its implementation. In addition, other scientists will not trust either the team or the results delivered, which might affect not only the single scientist in a collaboration but also his/her institution and/or students and the other collaborators.

\section{Conclusions}

Collaborative research in the modern world is becoming the standard. But, as confidence in the communication media is suffering from the rise of "fake news" and the poor quality of large amount of news and data shared on the social networks, to avoid a similar fate for research results, it is vital that collaboration in research goes hand in hand with quality in research.

Luckily the awareness of the importance of fostering high research quality and rigour is emerging worldwide. In Europe, The IMI "European Quality in Preclinical Data" (EQIPD) project will deliver simple recommendations to facilitate data quality without affecting innovation in the challenging field of preclinical neuroscience research. Cornerstone of the work is agreeing across stakeholders on the key variables influencing quality and delivering a prospectively validated consensus quality management system. EQIPD will importantly foster education and training 
on the principles and application of quality and rigour via an online educational platform providing certified education and training (EQIPD Consortium 2018). In the United States, there is a strong attention to rigour and quality in research, and the National Institute for Health has developed a specific guidance for grantees (National Institute for Health 2018), while the National Heart, Lung and Blood Institute has published the "Study Quality Assessment Tools" resource (National Heart, Blood and Lung Institute 2017). Thus, there are several resources available (only few mentioned above), and more are emerging to help researchers deliver excellent, robust research outputs of the highest impact. However, it is important that all efforts are coordinated and aligned globally: there is still an issue of fragmentation. In addition, while the effort of the EQIPD project represents a first important European effort to foster rigour and quality in research, European researchers still lack a common guidance. To be effective such guidance must be agreed across countries and be applicable both in national and international/Europe-wide collaborations.

The upcoming Framework programme Horizon Europe has the specific objective "to support the creation and diffusion of high-quality new knowledge, skills, technologies and solutions to global challenges" (Dalli 2018). To make this objective a reality, stakeholders in research and development in Europe and beyond have to put in place an aligned and effective policy for good research practice, in a collaborative way.

\section{References}

Accelerating Medicines Partnership AMP (2014) https://www.nih.gov/research-training/ accelerating-medicines-partnership-amp. Accessed 20 Feb 2019

Autism Innovative Medicine Studies-2-Trials AIMS-2-Trials Project (2018). https://www.aims-2trials.eu. Accessed $14 \mathrm{Feb} 2019$

Baron R, Maier C, Attal N et al (2017) Peripheral neuropathic pain: a mechanism-related organizing principle based on sensory profiles. Pain 158(2):261-272

Be The Cure" BTCure Project (2012) http://btcure.eu. Accessed 12 Feb 2019

Boehnke K, Iversen PW, Schumacher D et al (2016) Assay establishment and validation of a highthroughput screening platform for three-dimensional patient-derived colon cancer organoid cultures. J Biomol Screen 21(9):931-941

BTCure Consortium (2012) WP1 standardization workshop - summary for external communication. Available via BTCure. http://btcure.eu/wp-content/uploads/2012_03_26-BTCure-WP1workshop-summary-final_incl_images.pdf

Cell Assay Datasets (2017) UltraDD project. https://www.ultra-dd.org/tissue-platforms/cell-assaydatasets. Accessed 14 Feb 2019

Combatting Bacterial Resistance in Europe" COMBACTE Project (2015) https://www.combacte. com/about/about-combacte-net-detail/0. Accessed 12 Feb 2019

Critical Path Institute C-Path (2005) https://c-path.org/about/. Accessed 20 Feb 2019

Hubert Dalli (2018) The horizon Europe framework programme for research and innovation 2021-2027. Available via EPRS. http://www.europarl.europa.eu/RegData/etudes/BRIE/2018/ 627147/EPRS_BRI(2018)627147_EN.pdf. Accessed 20 Feb 2019 
DDMoRe Model Repository (2017) DDMore. http://repository.ddmore.eu. Accessed 12 Feb 2019

de Kraker M, Sommer H, de Veide F et al (2018) Optimizing the design and analysis of clinical trials for Antibacterials against multidrug-resistant organisms: a white paper from COMBACTE's STAT-net. Clin Infect Dis 67(12):1922-1931

Diabetes Research on Patient Stratification DIRECT Project (2013) www.direct-diabetes.org. Accessed 12 Feb 2019

Drug Disease Model Resources DDMoRe Project (2012) www.ddmore.eu. Accessed 12 Feb 2019

ENCODE Consortium (2017) Data standards. Available via ENCODE. https://www.encodeproject. org/data-standards. Accessed 12 Feb 2019

Encyclopedia of DNA Elements (ENCODE) (2019) https://www.genome.gov/10005107/theencode-project-encyclopedia-of-dna-elements. Accessed 12 Feb 2019

EQIPD Consortium (2018) EQIPD E-learning programme, preliminary version December 2018. Available via EQIPD https://quality-preclinical-data.eu/our-findings/training/. Accessed $20 \mathrm{Feb}$ 2019

eTRIKS/ELIXIR-LU - IMI Data Catalogue (2015) https://datacatalog.elixir-luxembourg.org/ about. Accessed 16 Feb 2019

European Autism Interventions - A Multicentre Study for Developing New Medications Project EU-AIMS (2012) https://www.eu-aims.eu. Accessed 12 Feb 2019

European Commission (2014) OpenAIRE initiative of the European Framework Programme H2020. Available via OPENAIRE. https://www.openaire.eu. Accessed 14 Feb 2019

European Federation of Pharmaceutical Industries and Associations (2015) EFPIA IMI2 consortium agreement template. Available via EFPIA. https://efpia.eu/media/.../efpia-model-consor tium-agreement-for-imi2-actions-2.docx. Accessed 14 Feb 2019

European Medicines Agency (2015) Letters of support EU-AIMS. Available via EMA. https:// www.ema.europa.eu/en/search/search?search_api_views_fulltext=EU-AIMS. Accessed $20 \mathrm{Feb} 2019$

European Medicines Agency (2016) Clinical development of medicinal products intended for the treatment of pain. Available via EMA. https://www.ema.europa.eu/en/clinical-developmentmedicinal-products-intended-treatment-pain. Accessed 20 Feb 2019

Faure JE, Dylagg T, Norstedt I et al (2018) The European innovative medicines initiative: progress to date. Pharm Med 32:243-249

Geissler J, Ryll B, Leto di Priolo S et al (2017) Improving patient involvement in medicines research and development: a practical roadmap. Ther Innov Reg Sci 51(5):612-619

Goldman M (2012) The innovative medicines initiative: a European response to the innovation challenge. Clin Pharmacol Ther 91(3):418-425

Goldman M, Seigneuret N, Eichler HG (2015) The innovative medicines initiative: an engine for regulatory science. Nat Rev Drug Dis 14:1-2

Grossman RL (2017) An introduction to the brain commons. Available via Cohen Veterans Bioscience. https://www.cohenveteransbioscience.org/2017/09/27/an-introduction-to-thebrain-commons. Accessed 14 Feb 2019

Holmdahl R (2015) Animal models for rheumatoid arthritis Available via HsTalks. https://hstalks. com/t/3076/animal-models-for-rheumatoid-arthritis/?biosci. Accessed 12 Feb 2019

Laverty H and Poinot M (2014) IP policy forum: intellectual property rights (IPR) in collaborative drug development in the EU: helping a European public-private partnership deliver - the need for a flexible approach to IPR. 18 Marq. Intellectual Property L Rev 31. Available via Marquette University. https://scholarship.law.marquette.edu/iplr/vol18/iss1/19. Accessed 14 Feb 2019

Majumder MA, Cook-Deegan R, McGuire AL (2016) Beyond our borders? Public resistance to global genomic data sharing. PLoS Biol 14(11):e2000206

Mascalzoni D et al (2015) International Charter of principles for sharing bio-specimens and data. Eur J Hum Genet 23:721-728

Mechanism-Based Integrated Systems for the Prediction of Drug-Induced Liver Injury MIP-DILI Project (2012) http://www.mipdili.eu. Accessed 12 Feb 2019 
Methods for Systematic Next Generation Oncology Biomarker Development ONcoTRACK Project (2016) http://www.oncotrack.eu/home/index.html. Accessed 12 Feb 2019

National Heart, Blood and Lung Institute (2017) Study quality assessment tools. Available via NHBLI. https://www.nhlbi.nih.gov/health-topics/study-quality-assessment-tools. Accessed $20 \mathrm{Feb} 2019$

National Institute for Health (2018) Guidance: rigor and reproducibility in grant applications. Available via NIH. https://grants.nih.gov/policy/reproducibility/guidance.htm. Accessed $20 \mathrm{Feb} 2019$

Nature Editorial (2017) On data availability, reproducibility and reuse. Nat Cell Biol 19:259

Nosek BA et al (2015) Promoting an open research culture. Science 348(6242):1422-1425

Open Access and Data Management for Projects (2015) Available via the innovative medicines initiative. https://www.imi.europa.eu/resources-projects/open-access-and-data-managementprojects. Accessed 12 Feb 2019

Parse Insight Consortium (2019) Insight into digital preservation of research outputs in Europe. Available via Liber europe. https://libereurope.eu/wp-content/uploads/2010/01/PARSE. Insight.-Deliverable-D3.4-Survey-Report.-of-research-output-Europe-Title-of-Deliverable-Sur vey-Report.pdf. Accessed 14 Feb 2019

Sanz F et al (2017) Legacy data sharing to improve drug safety assessment: the eTOX project. Nat Rev Drug Discov 16:812

Schütte M, Risch T, Abdavi-Azar N et al (2017) Molecular dissection of colorectal cancer in pre-clinical models identifies biomarkers predicting sensitivity to EGFR inhibitors. Nat Commun 10(8): 14262

Sison-Young RL, Lauschke VM, Johannet E et al (2017) A multicenter assessment of single-cell models aligned to standard measures of cell health for prediction of acute hepatotoxicity. Arch Toxicol 91(3): 1385-1400

Stem Cells for Biological Assays of Novel Drugs and Predictive Toxicology Project (2014) STEMBANCC. https://stembancc.org. Accessed 12 Feb 2019

Teare HJA, de Masi F, Banasik K et al (2018) The governance structure for data access in the DIRECT consortium: an innovative medicines initiative (IMI) project. Life Sci Soc Policy 14:20

Tenopir C, Allard S, Douglass K et al (2011) Data sharing by scientists: practices and perceptions. PLoS One 6(6):e21101

The Innovative Medicines Initiative. (2009) https://www.imi.europa.eu. Accessed 12 Feb 2019

The Innovative Medicines Initiative (2017a) 'More successful than what we thought possible' - an interview with the Europain project coordinator. Available via IMI https://www.imi.europa.eu/ projects-results/success-stories-projects/more-successful-what-we-thought-possible-interview. Accessed 21 Feb 2019

The Innovative Medicines Initiative (2017b) Guidelines on engaging with regulators. Available via IMI. https://www.imi.europa.eu/resources-projects/guidelines-engaging-regulators. Accessed $20 \mathrm{Feb} 2019$

The Innovative Medicines Initiative Radical Collaborations (2018) Available via IMI. https://www. imi.europa.eu/projects-results/success-stories-projects/radical-collaboration-shaking-pharma ceutical-industry. Accessed 12 Feb 2019

Trifan A et al (2018) A methodology for fine-grained access control in exposing biomedical data. In: Building continents of knowledge in oceans of data: the future of co-created eHealth series studies health technology and informatics, vol 247. IOS Press, Amsterdam, pp 561-565

Unrestricted Leveraging of Targets for Research Advancement and Drug Discovery ULTRADD Project (2016) https://www.ultra-dd.org. Accessed 12 Feb 2019

Vollert J, Mainka T, Baron R et al (2015) Quality assurance for quantitative sensory testing laboratories: development and validation of an automated evaluation tool for the analysis of declared healthy samples. Pain 156(12):2423-2430 
Volpato V, Smith J, Sandor C et al (2018) Reproducibility of molecular phenotypes after long-term differentiation to human iPSC-derived neurons: a multi-site omics study. Stem Cell Rep 11 (4):897-911

Wicherts JM, Bakker M, Molenaar D (2011) Willingness to share research data is related to the strength of the evidence and the quality of reporting of statistical results. PLoS One 6(11): e26828

Wilkinson MD, Dumontier M, Aalbersberg IJ et al (2016) The FAIR guiding principles for scientific data management and stewardship. Sci Data 3:160018

Open Access This chapter is licensed under the terms of the Creative Commons Attribution 4.0 International License (http://creativecommons.org/licenses/by/4.0/), which permits use, sharing, adaptation, distribution and reproduction in any medium or format, as long as you give appropriate credit to the original author(s) and the source, provide a link to the Creative Commons licence and indicate if changes were made.

The images or other third party material in this chapter are included in the chapter's Creative Commons licence, unless indicated otherwise in a credit line to the material. If material is not included in the chapter's Creative Commons licence and your intended use is not permitted by statutory regulation or exceeds the permitted use, you will need to obtain permission directly from the copyright holder. 